\title{
Soft tissue handling during implant placement
}

\author{
What are the best techniques for handling soft tissues during dental \\ implant placement?
}

\author{
Esposito M, Grusovin MG, Maghaireh H, Coulthard P, \\ Worthington HV. \\ Interventions for replacing missing teeth: management of soft tis- \\ sues for dental implants. Cochrane Database Syst Rev 2007; issue 3
}

Data sources The Cochrane Oral Health Group's Trials Register, Cochrane Central Register of Controlled Trials, Medline and Embase were searched. Handsearching included several dental journals. Authors of all identified trials, an internet discussion group and 55 dental implant manufacturers were contacted to find unpublished randomised controlled trials (RCT).

Study selection All RCT of root-form osseo-integrated dental implants comparing various techniques to handle soft tissues when placing dental implants were included. Outcome measures considered were: prosthetic and implant failures, aesthetics evaluated by patients and dentists, biological complications, postoperative pain, patient preference, ease of maintenance by patient, and width of the attached or keratinised mucosa.

Data extraction and synthesis Screening of eligible studies, assessment of the methodological quality of the trials and data extraction were conducted in duplicate and independently by two review authors. Authors were contacted for missing information. Results were expressed as random-effects models using mean differences for continuous outcomes, and risk ratios for dichotomous outcomes, with $95 \%$ confidence intervals. Heterogeneity was to be investigated, including both clinical and methodological factors.

Results Eight RCT were identified and five trials, which included 140 patients in total, were selected. Two trials (comprising 100 patients) compared flapless placement of dental implants with conventional flap elevation, two trials (20 patients) crestal versus vestibular incisions. On a patient (rather than per-implant) basis, implants placed with a flapless technique and implant exposures performed with a laser induced significantly less postoperative pain than flap elevation. There were no statistically significant differences for any of the other analyses.

Conclusions Flapless implant placement is feasible and has been shown to reduce patient postoperative discomfort in appropriately selected patients. Sufficient, reliable evidence is not available for recommendations to be made about which techniques or materials for incision or suture are most beneficial to patients. This is also the case for techniques to correct or augment perimplant soft tissues or to increase the width of keratinised or attached mucosa. Properly designed and conducted RCT are needed to provide reliable answers to these questions.

Address for correspondence: Luisa Fernandez, Oral Health Group Coordinator, MANDEC 3rd Floor Dental Hospital, University of Manchester, Higher Cambridge Street, Manchester M15 6FH, UK. E-mail: luisa.fernandez@manchester.ac.uk

\section{Commentary}

The importance of the soft tissue-implant interface has rarely been the focus of studies, even though it is a vital element of treatment, especially when there are aesthetic considerations. Implant placement in a suitably indicated site is usually considered an irreversible procedure, where the first bite of the cherry is always the optimum and needs to be planned properly. The manipulation of soft tissues around dental implants requires an understanding of the biology and anatomy of the periodontium, with soft tissue biotype influencing possible complications. Experienced clinical judgement and scanning investigations in appropriately selected patients play a vital role in the prognosis and likely outcome, as do patient factors such as plaque control, smoking and periodontal susceptibility.

Several questions were put forward in this Cochrane review. The five studies included related to the need for flap elevation and the most appropriate flap design. There were no available trials, however, that concentrated on soft tissue correction or augmentation techniques, on techniques to increase the perimplant attached or keratinised mucosa, or on suturing techniques.

In total, 370 implants in 140 patients were included. The statistical unit was the patient rather than the implant. Two studies (total, 100 patients) were included when the question addressed whether flap elevation was necessary. One study compared conventional flap design with a minimally invasive design (6-day review) and the other compared flapless with conventional flap designs (3-year review). These concluded that flapless and minimally invasive surgery results in reduced postoperative pain and oedema. In the latter study, the implants placed without raising a flap were loaded immediately and the conventionally placed implants were loaded 3-4 months later. These two studies did not look at implant or prosthetic failures.

Three studies were included that considered the best flap design. Two of these studies (total of 20 patients) compared crestal and vestibular flaps. There was no statistical difference between their outcome measures. The final study (10 patients) compared the use of laser for implant exposure with conventional incisions. Interestingly, only two out of the 10 patients who had implant exposure with the laser required local anaesthesia, which may have significant implications for patient comfort. This review revealed that some evidence supports a place for flapless surgical implant treatment and the use of laser implant exposure, based purely on patient comfort, although no mention was made of the thickness of tissue incision or the use of analgesia. The literature clearly still lacks studies with adequate long-term follow-up. Large patient-base comparative techniques in multicentre studies may be a future research opportunity.

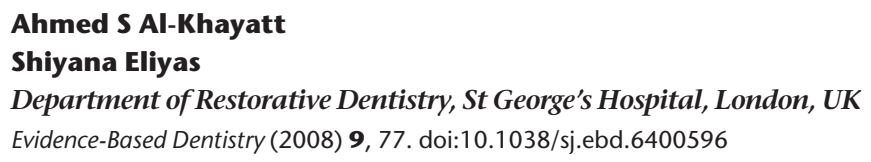

